\title{
Análise do perfil de uso de narguilé entre estudantes de medicina
}

\author{
Analysis of narguille use profile among medicine students \\ Análisis del perfil de uso de narguille en estudiantes de medicina
}

Stéphanie Cândida Abdala Gomes ${ }^{1 *}$, Ana Vitória Costa Braga ${ }^{1}$, Camila Gomes Vieira ${ }^{1}$, João Nascimento Mendonça Neto ${ }^{1}$, Lucas Lourenço Almeida ${ }^{1}$, Lídia Acyole de Souza², Milena Moreira Lima1.

\begin{abstract}
RESUMO
Objetivo: Analisar o perfil de uso de narguilé entre acadêmicos do curso de medicina da região central goiana. Métodos: Estudo epidemiológico, analítico, transversal e quantitativo, com universitários do $1^{\circ}$ ao 8ำ período. Coleta de dados por questionários com variáveis sociodemográficas, socioeconômicas e caracterização do uso de narguilé pelo participante. Foi utilizado análise estatística com software adotando um nível de significância de $5 \%$ ( $p$ <0.05). Resultados: A prevalência do uso de narguilé foi de $51,3 \%$, predominantemente por: homens $(57,4 \%)$, faixa etária de $\geq 30$ anos $(57,1 \%)$, solteiros $(51,5 \%)$ e de classe econômica C1 (37\%). A maior frequência declarada de uso esporádico (79,2\%) apresentando efeitos colaterais (56\%) e motivação de uso por influência (45,6\%). Cerca de 19,2\% não acreditam no aconselhamento profissional para a queda do tabagismo, mas $97,7 \%$ são a favor da orientação. Conclusão: A pesquisa demonstra que o público masculino, acima de 30 anos, solteiro e de classe econômica $\mathrm{C} 1$ foi predominante no uso do narguilé. Portanto, é imprescindível a análise da influência da inserção na universidade como motivador para o uso do dispositivo, além de investigar quais medidas podem ser tomadas para combater a manutenção desse hábito, que é majoritária e preocupante.
\end{abstract}

Palavras-chave: Tabaco, Narguilé, Universitários, Nicotina.

\begin{abstract}
Objective: To analyze the profile of hookah use among medical students in the central region of Goiás. Methods: Epidemiological, analytical, transversal and quantitative study, with university students from the 1st to the 8th period. Data collection through questionnaires with sociodemographic and socioeconomic variables and characterization of the participant's use of hookah. Statistical analysis was used with software adopting a significance level of $5 \%(p<0.05)$. Results: The prevalence of hookah use was $51.3 \%$, predominantly by: men $(57.4 \%)$, aged $\geq 30$ years $(57.1 \%)$, single $(51.5 \%)$ and economic class $\mathrm{C} 1(37 \%)$. The highest reported frequency of sporadic use $(79.2 \%)$ had side effects $(56 \%)$ and use was motivated by influence $(45.6 \%)$. About $19.2 \%$ do not believe in professional advice for smoking cessation, but $97.7 \%$ are in favor of counseling. Conclusion: The research demonstrates that the male audience, over 30 years old, single and from the $\mathrm{C} 1$ economic class, was predominant in the use of the hookah. Therefore, it is essential to analyze the influence of admission to the university as a motivator for the use of the device, in addition to investigating what measures can be taken to combat the maintenance of this habit, which is a major and worrisome habit.
\end{abstract}

Keywords: Tobacco, Hookah, University students, Nicotine.

\section{RESUMEN}

Objetivo: Analizar el perfil del uso de narguiles entre estudiantes de medicina de la región central de Goiás. Métodos: Estudio epidemiológico, analítico, transversal y cuantitativo, con estudiantes universitarios del $1^{\circ}$ al 8o período. Recolección de datos mediante cuestionarios con variables sociodemográficas y socioeconómicas y caracterización del uso de narguile por parte de los participantes. El análisis estadístico se utilizó con un software que adoptó un nivel de significancia del $5 \%(p<0,05)$. Resultados: La prevalencia del uso de narguile fue del $51,3 \%$, predominantemente por: hombres $(57,4 \%)$, edad $\geq 30$ años $(57,1 \%)$, solteros $(51,5 \%)$ y clase económica C1 (37\%). La frecuencia más alta informada de uso esporádico $(79,2 \%)$ tuvo efectos secundarios

${ }^{1}$ Universidade Evangélica de Goiás (UniEVANGÉLICA), Anápolis - GO.

*E-mail: stephanie abdala@hotmail.com

2 Centro Universitário Estácio de Goiás, Goiânia - GO.

SUBMETIDO EM: 10/2021 
(56\%) y el uso estuvo motivado por la influencia (45,6\%). Aproximadamente el $19,2 \%$ no cree en el asesoramiento profesional para dejar de fumar, pero el 97,7\% está a favor del asesoramiento. Conclusión: La investigación demuestra que el público masculino, mayor de 30 años, soltero y de la clase económica C1, fue predominante en el uso del narguile. Por tanto, es fundamental analizar la influencia del ingreso a la universidad como motivador del uso del dispositivo, además de investigar qué medidas se pueden tomar para combatir el mantenimiento de este hábito, que es un hábito importante y preocupante.

Palabras-clave: Tabaco, Narguile, Universitarios, Nicotina.

\section{INTRODUÇÃO}

Um dos tópicos mais assombrosos para a pneumologia e para a saúde pública em geral é quanto ao uso do tabaco, haja vista que o mesmo precede diversas patologias, além de ser uma importante causa de perda da qualidade de vida e de vício. No Brasil, tornou-se uma prática muito comum após contato entre colonos e colonizadores, pois estes, vindos da Europa, construíram e embelezaram esse hábito por meio de propagandas/filmes/telenovelas e o correlacionaram de diversas formas com charme, status e poder econômico. Atualmente, observa-se que o tabagismo habitual está em queda, por meio da redução do uso dos cigarros tradicionais, por outro lado, deve-se salientar que isso só representa uma mudança de padrão do uso de tabaco, visto que os métodos não convencionais vêm ganhando grande espaço no mercado, tais como, cigarro eletrônico, cachimbo, charuto e narguilé (MALTA DC, 2020).

O tabagismo está enraizado na sociedade brasileira desde antes da colonização (FONSECA A, 2017). Mas, perpassando o status social advindo do hábito de fumar e a disseminação dessa prática no mundo, ficou cada vez mais evidente que as comorbidades causadas pelo tabaco superaram os benefícios oferecidos por essa cultura. Atualmente, segundo dados da OMS, o tabaco mata mais de 8 milhões de pessoas por ano no mundo, sendo que somente no Brasil, 428 pessoas morrem por dia por causa da dependência a nicotina (princípio ativo presente no tabaco e causador da dependência física, químicas entre outros malefícios) (WORLD HEALTH ORGANIZATION, 2020).

Dado os conhecidos malefícios do tabaco, e seu impacto na saúde pública e econômica do país, desde 1960, o Brasil tem condicionado a venda do cigarro à publicidade com advertências sobre o risco à saúde associado ao tabaco (TEIXEIRA L e JAQUES T, 2021). E desde então, diversas políticas públicas foram desenvolvidas contra o tabagismo, e mais especificamente relacionadas ao consumo de tabaco via cigarro (REIS AAC, et al., 2018).

Nesse cenário brasileiro atual de forte combate ao uso do cigarro, as formas não convencionais de tabaco ganham espaço e tornam-se mais fáceis e despercebidamente mais disseminadas. Dentre tais alternativas nota-se o narguilé, que foi criado na Índia no século XVI, sendo considerado um método tradicional na África, Ásia e especialmente na região do Mediterrâneo Oriental. E que, mais recentemente, tem se disseminado e criado raízes em países ocidentais (MAZIAK W, et al., 2019; MARTINS RS e SANTOS UP, 2019).

Também chamado de shisha, hookah ou waterpipe, o narguilé é uma forma de fumo em que o tabaco é aquecido e a fumaça gerada passa por um recipiente de água para depois ser aspirada por meio de uma mangueira. Desde sua invenção, tem-se a crença de que a água atuaria como um filtro e, assim, o narguilé seria menos danoso para o fumante. Essa ideia errônea somada às inovações tecnológicas trazidas nesses dispositivos e à possibilidade de adição de sabores e aromas, transfigura o narguilé como um grande atrativo social, frequentemente compartilhado entre amigos em casa, boates, bares e cafés, propagando seu uso com mais celeridade (RIBEIRO M e CRUZ RS, 2016; MALTA DC, et al., 2018; INSTITUTO NACIONAL DE CÂNCER (INCA), 2019).

No Brasil, apesar de dados epidemiológicos ainda serem escassos, há evidências que afirmam a rápida disseminação de narguilé, em especial entre os jovens (WARREN CW, et al., 2018). Esse fato gera grande preocupação para estudiosos e trabalhadores da área da saúde, haja vista que esses produtos, sobretudo 0 narguilé, são amenizados pelas mídias e servem como "porta de entrada" entre a juventude para a introdução no consumo do tabaco e suas formas, inclusive do cigarro convencional (SAADE G, et al., 2019; TAMIM H, 
et al., 2020). Um estudo realizado por Oliveira LAS (2016), na cidade de Anápolis-GO, evidenciou que o uso de narguilé entre estudantes de uma instituição de ensino foi de 47,32\%. Já em 2019, em Goiânia - GO, outro estudo apontou que dos estudantes de medicina entrevistados 59,6 \% já experimentaram narguilé (ARAúJO RS, et al., 2019).

Neste sentido, com o intuito de diminuir os índices de tabagismo ou o consumo de derivados de tabaco no Brasil, no Dia Nacional do Combate ao Fumo em 2015, o Ministério da Saúde realizou uma campanha tendo como tema principal o narguilé, com o objetivo de alertar sobre as principais consequências e malefícios do uso de tabaco para o organismo (INCA), 2015; REIS AAC, et al., 2018). Além disso, o Ministério da Saúde juntamente com a Atenção Primária em Saúde, tem contribuído satisfatoriamente com as diversas ações educativas que minimizam o consumo indiscriminado de tabaco, principalmente entre os jovens (TEODORO WR, 2019).

Como justificativa, os esforços legais para se limitar o tabagismo no Brasil têm contribuído para o declínio do consumo do tabaco entre a sociedade no geral, entretanto, conforme estudos de Monteiro CA, et al. (2007), existe uma faixa etária brasileira que caminha na contramão dessa estatística. Segundo Pesquisa Nacional de Saúde do Escolar (PeNSE) realizada pelo Instituto Brasileiro de Geografia e Estatística (IBGE), em 2009, 24\% dos adolescentes de 13 a 15 anos haviam experimentado cigarros. Já na PeNSE de 2012, esse percentual alcançava 30\% dos jovens entrevistados (BARRETO SM, et al., 2018). O que demonstra como é relevante entender os motivos para os quais jovens inseridos em instituições de ensino ainda permaneçam sustentando práticas tabagistas enquanto o mundo tenta minimizá-la (PEREIRA JS, et al., 2018).

Há um consenso entre estudiosos que justificam esse contraste em relação aos jovens e o restante da população. Infere-se que jovens ingressantes em universidades são expostos a difíceis adaptações, deixando-os vulneráveis à manutenção do fumo. Assim como há uma propensão maior para se fumar entre os jovens que convivem com familiares fumantes ou que não são inibidos por fumarem no cenário domiciliar (BARRETO SM, et al., 2018; WERNECK FDA, et al., 2018).

Percebe-se, então, que inúmeras são as facilidades para a juventude aderir ao tabagismo e seria ingenuidade acreditar que essa adesão acontece apenas para cigarros convencionais. Com as dificuldades para comercialização do cigarro, a indústria investe em novas formas de tabaco vendendo a ideia de que é menos prejudicial à saúde (SANTOS PU, 2018). Segundo o Ministério da Saúde, devido às medidas restritivas ao cigarro, o narguilé tem sido amplamente disseminado entre essa faixa etária (BRASIL, 2011). Nessa modalidade, a composição não é padronizada e o percentual de nicotina pode ser de $2 \%$ a $3 \%$ mais elevado do que em cigarros comuns (BRASIL, 2011; SANTOS PU, 2018).

É extremamente importante conhecer qual a prevalência de jovens universitários que utilizam essa forma alternativa do tabaco: o narguilé. Logo, a presente pesquisa, ao trazer dados que amplificam o conhecimento epidemiológico sobre o uso de narguilé, auxiliará em políticas públicas, de modo que haja um melhor incentivo à promoção e manutenção da saúde. Sendo assim, este trabalho teve como objetivo principal analisar o perfil de uso de narguilé entre acadêmicos do curso de medicina da região central goiana no ano de 2021.

\section{MÉTODOS}

Trata-se de uma pesquisa epidemiológica, analítica transversal, descritiva e de análise quantitativa. A pesquisa foi desenvolvida por meio da utilização de dados coletados através de questionários estruturados pela Associação Brasileira de Empresas de Pesquisa (ABEP) e pela Global Health Professions Student Survey.

As variáveis analisadas para o estudo foram: dados sociodemográficos (sexo, idade, estado civil, período de curso, cidade de moradia, hábitos de vida), dados socioeconômicos (quantidade de veículos, empregados mensalistas, eletrodomésticos, cômodos de residência e nível educacional) e dados sobre a experiência e uso do narguilé.

Os critérios de inclusão neste estudo foram: ser acadêmico regular e matriculado no curso de medicina da região central goiana, do 1ำ ao $8^{\circ}$ período; Ser maior de 18 anos de idade; Preencher adequadamente os 
questionários usados para a coleta de dados. Os critérios de exclusão foram: Acadêmico de medicina da região central goiana do $9^{\circ}$ ao $12^{\circ}$ período; Questionários incompletos; Não aceitação do Termo de Consentimento Livre e Esclarecido (TCLE).

Para a tabulação dos dados e análise estatística descritiva, com frequência relativa e absoluta, foi utilizado o Microsoft Office Excel e para a elaboração da tabela e gráficos Word 2016.

O projeto foi submetido seguindo a Resolução 466/2012 do Conselho Nacional de Saúde (CNS), que dispõe sobre pesquisas com seres humanos e foi aprovado pelo Comitê de Ética em Pesquisa da Universidade Evangélica Goiás (UniEVANGÉLICA) de parecer n.ำ 4.665.021.

\section{RESULTADOS}

Dentre os 765 estudantes, obteve-se 540 participantes da pesquisa. Destes, 35 questionários foram excluídos devido a erros de preenchimento $(n=4)$; participantes menores de idade $(n=13)$; e questionários incompletos $(n=18)$. Portanto, a amostra analisada totalizou 505 acadêmicos do $1^{\circ}$ ao $8^{\circ}$ período do curso de medicina da região central goiana. As prevalências e os números são apresentados para a população de pesquisa e, em seguida, são desagregadas por sexo, faixa etária, estado civil, local de residência e com quem reside (Tabela $\mathbf{1}$ ).

Dentre os 505 questionários respondidos e válidos, foi identificado que a prevalência do uso de narguilé foi de $51,3 \%$, sendo predominantemente utilizado por: homens $(57,4 \%)$, faixa etária de $\geq 30$ anos $(57,1 \%)$, solteiros (51,5\%), de classe econômica C1 (37\%) e moradores de outros municípios (53,8\%) (Tabela 1).

A associação entre o uso do narguilé e as variáveis sociodemográficas e econômicas apresentou uma tendência de correlação quando analisado o qui-quadrado, sobretudo quanto à classe econômica $(p=0,029)$, onde $37 \%$ dos participantes da classe $\mathrm{C} 1$, com renda média domiciliar de 2.409,01 reais, alegaram usar 0 narguilé e à idade $(p=0,034)$, com maior prevalência de uso entre a faixa etária superior ou igual à 30 anos $(57,1 \%)$. Nota-se ainda que aqueles que declararam residir com os pais tendem a usar menos narguilé $(45,3 \% ; p=0,055)$, quando comparados aos demais que residem sozinhos $(56,8 \%)$, em repúblicas $(57,7 \%)$ ou em outras modalidades (60\%) (Tabela 1).

Tabela 1 - Perfil sociodemográfico dos acadêmicos de medicina, total e estratificado entre os participantes da pesquisa da região central goiana, 2021.

\begin{tabular}{lccc}
\hline \multicolumn{4}{c}{ USO DE NARGUILÉ n (\%) } \\
\hline Variável & Sim & Não & Teste Qui-quadrado (p) \\
\hline Sexo & & & \\
\hline Feminino & $166(48,4)$ & $177(51,6)$ & 0,058 \\
Masculino & $93(57,4)$ & $69(42,6)$ & \\
\hline Idade & & & \\
\hline $18-19$ & $52(41,3)$ & $74(58,7)$ & 0,034 \\
$20-29$ & $203(54,6)$ & $169(45,4)$ & \\
$\geq 30$ & $4(57,1)$ & $3(42,9)$ & 0,557 \\
\hline Estado Civil & & & \\
\hline Solteiro & $253(51,5)$ & $238(48,5)$ & \\
Casado & $5(50)$ & $5(50)$ & \\
Divorciado & $1(25)$ & $3(75)$ & \\
\hline Com quem reside & & & \\
\hline Pais & $117(45,3)$ & $141(54,7)$ & \\
República & $15(57,7)$ & $11(42,3)$ & \\
Sozinho & $100(56,8)$ & $76(43,2)$ & \\
Outros & $27(60)$ & $18(40)$ & \\
\hline Residência & $117(45,3)$ & $141(54,7)$ & \\
\hline Goiânia & $83(53,2)$ & $73(46,8)$ & \\
Anápolis & $169(50,3)$ & $167(49,7)$ & \\
Outras & $7(53,8)$ & $6(46,2)$ & \\
\hline
\end{tabular}

Fonte: Gomes SCA, et al., 2021. 
Para identificar o perfil do uso atual de narguilé, os estudantes foram questionados sobre a presença de sabor e aroma, o que os motivaram a experimentá-lo, presença de efeitos colaterais devido ao uso e qual a frequência de uso atual. Adicionalmente, ao questionar sobre a idade do primeiro contato e uso de narguilé, a maioria dos estudantes $(56,3 \%)$ relatou o primeiro contato com o produto antes dos 18 anos (Tabela 2 ).

Tabela 2 - Caracterização do modo de utilização do narguilé entre os acadêmicos de medicina, da região central goiana, 2021.

\begin{tabular}{lcc}
\hline Variável & $\mathbf{n}$ & $\%$ \\
\hline Motivo do uso & 110 & 42,5 \\
Curiosidade & 19 & 7,3 \\
Diversão & 118 & 45,6 \\
Influência & 12 & 4,6 \\
Outras & 4 & 1,5 \\
\hline Frequência de uso & 5 & 1,9 \\
Diário & 10 & 3,9 \\
Semanal & 205 & 79,2 \\
Mensal & 35 & 13,5 \\
Esporádico & & \\
Parou de usar & 11 & 4,2 \\
\hline Sabor/Aroma & 40 & 15,4 \\
Sem sabor & 48 & 18,5 \\
Morango & 73 & 28,2 \\
Melancia & 87 & 33,6 \\
Menta & & \\
Outros & 145 & 56 \\
\hline Efeitos Colaterais & 114 & 44 \\
Sim & & \\
Não & & \\
\hline
\end{tabular}

Fonte: Gomes SCA, et al., 2021.

Quanto aos hábitos de vida dos participantes que utilizam narguilé, as variáveis "atividade física", "reside com fumante" e "fumantes na família" estão demonstradas na Tabela 3, cuja análise demonstra correlações significativas entre o uso de narguilé e as duas últimas variáveis, ambas com $p=0,004$. Nota-se que 0 percentual de participantes usuários de narguilé que reside com fumante $(14,3 \%)$ ou tem fumante na família (34\%) é superior àquele encontrado entre os participantes que alegaram não usar narguilé.

Tabela 3 - Hábitos de vida dos acadêmicos de medicina da região central goiana, 2021.

\begin{tabular}{lccc}
\hline \multicolumn{4}{c}{ USO DE NARGUILÉ $\mathbf{n}(\%)$} \\
\hline Variável & Sim & Não & Teste Qui-quadrado (p) \\
\hline Atividade Física & & & \\
\hline Sim & $181(69,9)$ & $172(69,9)$ & \multirow{2}{*}{0,093} \\
Não & $78(30,1)$ & $74(30,1)$ & \\
\hline Reside com fumante & & & \multirow{2}{*}{0,004} \\
\hline Sim & $37(14,3)$ & $16(6,5)$ & \\
Não & $222(85,7)$ & $230(93,5)$ & 0,004 \\
\hline Fumante na família & & & $54(22)$ \\
\hline Sim & $88(34)$ & $192(78)$ & \\
\hline
\end{tabular}

Fonte: Gomes SCA, et al., 2021.

No Gráfico 1 estão descritos os efeitos colaterais agudos alegados pelos participantes devido ao uso do narguilé. Observou-se, nesse sentido, a predominância da sensação de redução da pressão arterial em relação aos outros efeitos, abrangendo $66,9 \%$ dos relatos, seguido por tontura $(21,4 \%)$, e em menor proporção outros $(6,9 \%)$ e tosse $(4,8 \%)$. 
Gráfico 1 - Principais efeitos colaterais agudos declarados pelos participantes após o uso do dispositivo narguilé.

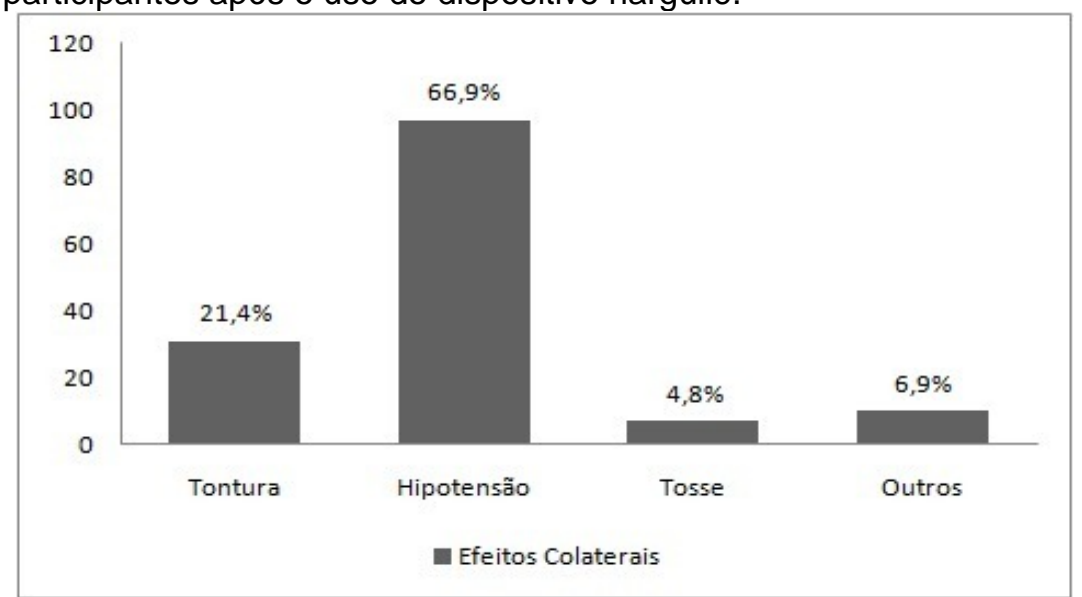

Fonte: Gomes SCA, et al., 2021.

\section{DISCUSSÃO}

Por meio do estudo, percebeu-se que os aromas, os sabores, a socialização e o mito de que são menos prejudiciais à saúde e que não causam dependência, são alguns fatores atrativos que foram apontados em diversos estudos recentes para o aumento da prevalência do narguilé nos últimos anos e que corroboram com os dados obtidos neste trabalho.

Em relação ao gênero, dentre os 259 acadêmicos, quase dois terços que declararam usar narguilé eram do sexo feminino. Contudo, o presente estudo teve como população analisada majoritariamente o sexo feminino, sendo necessária a análise de uso de narguilé estratificada intrasexo para a manutenção da fidedignidade do estudo. Assim, mesmo a quantidade de uso sendo maior entre as mulheres, tem-se que a frequência de uso entre o sexo feminino é menor em relação ao sexo masculino.

Um estudo realizado entre maio e dezembro de 2015 revelou que a prevalência de uso do narguilé está entre jovens de alto nível socioeconômico (BERTONI N, et al., 2019). Nesse sentido, pode-se correlacionar, em uma primeira avaliação, que o maior poder aquisitivo possibilita aos jovens o acesso com maior facilidade a esse tipo de tabaco. Entretanto, o presente estudo obteve dados discrepantes, aos quais demonstram que houve uma maior prevalência de uso de narguilé entre a classe C1. Diante dessa perspectiva, pode-se sobrelevar o fato de que isso representa um possível viés para a análise, haja vista que grande parte dos participantes saíram de suas casas muito jovens para fazer faculdade em outro município. Portanto, ao responderem aos questionários é provável que tenham considerado suas moradias alternativas em Anápolis, que, certamente, não refletem de forma fidedigna sobre toda a renda média familiar que esse indivíduo possui.

Outro ponto importante a ser discutido, acerca dos hábitos de vida dos participantes da pesquisa, é quanto à influência que a família e a residência do indivíduo podem ter sobre a decisão dele de utilizar ou não o narguilé e outras formas de tabaco. Neste trabalho, verificou-se que a frequência absoluta dos que utilizaram narguilé e não tinham fumantes na família foi quase o dobro quando comparado aos que fumam e possuem um membro de primeiro grau fumante. Contudo, ao analisar a variável intracategórica foi observado que há sim uma relação diretamente proporcional entre família e o uso de narguilé, uma vez que a maior parte dos participantes eram usuários de narguilé e tinham familiares também fumantes.

Além disso, a relação "reside com fumante" e "uso de narguilé" foi bastante significativa, considerando dois pontos fundamentais para o entendimento do estudo: 1. Mais de dois terços dos que usaram o dispositivo narguilé residiam com outros tabagistas; 2 . Mais da metade dos participantes que residiam em repúblicas ou em outros lugares eram usuários de narguilé. Nesse contexto, deve-se dizer que morar com os pais é um fator vantajoso e de resguardo diante desta problemática, pois a pesquisa revelou baixos índices de uso de narguilé entre os participantes que residiam com os familiares quando comparados às demais opções de moradias. 
Diante dessa perspectiva, vale salientar que muitos trabalhos foram ao encontro desse resultado, a exemplo do que foi demonstrado pelos autores Barrenechea MA, et al. (2017) e Horta BL, et al. (2021), que investigaram uma forte associação entre o hábito de fumar e a predisposição ao fumo pelos mais novos do núcleo familiar, sobretudo, na adolescência. Porém, deve-se ressaltar que há uma característica fundamental que diferencia esse trabalho dos demais analisados, que é justamente a associação com o narguilé, haja vista que, assim como a maioria das pesquisas científicas que envolvem o tabagismo, aquelas também não foram associadas diretamente com os novos dispositivos de tabaco, como o narguilé, mas somente com o cigarro tradicional, tendo esse componente como um viés para a comparação dos dados.

Todavia, ainda assim, ao analisar somente a correlação narguilé e influência familiar/residência, alguns pesquisadores, como Malta DC, et al. (2017) e Oliveira MA (2019), trazem nos seus estudos que a família e os amigos representam de fato uma forte influência, corroborando com os resultados obtidos nesta pesquisa.

No estudo de Oliveira MA (2019), por exemplo, eles representavam mais que $75 \%$ da influência para o uso de narguilé entre os acadêmicos de medicina da Universidade Estadual de Ponta Grossa. Dessa forma, torna-se claro que a influência, seja de amigos ou de familiares, identificado em quase metade dos participantes desta pesquisa, por meio da questão aberta do questionário "motivo de uso", condiz com os estudos recentes desse tema.

Quanto ao hábito de praticar atividades físicas, mais da metade do público que respondeu aos questionários afirmou realizar exercícios físicos regularmente. Dessa forma, essa questão trouxe um resultado benéfico e, portanto, se apresenta como um ótimo fator protetor para esses usuários, haja vista que em diversos estudos as variáveis respiratórias tiveram seus níveis rebaixados demonstrando diversos prejuízos às condições pulmonares, independente do produto do tabaco utilizado, ao contrário do que acreditam os tabagistas de narguilé (LUNELLI ML, et al., 2019; SZKLO AS, et al., 2021).

Outro atrativo ao uso de narguilé é a presença de sabor ou aroma, uma vez que, entre os acadêmicos que utilizam narguilé, a minoria alegou utilizar narguilé sem aroma ou sabor. De acordo com Tavares IB, et al. (2019), grande parte dos usuários de narguilé tem como motivação o gosto e o cheiro do narguilé. Assim, tendo em vista que o narguilé foi gradativamente sendo modernizado para maior facilidade durante o fumo e atratividade, tem-se que a adição de cores, sabores e aromas contribuem de fato para sua atual disseminação (ALMEIDA LM, et al., 2017).

Em relação ao questionamento acerca dos malefícios do narguilé, a maioria dos usuários desse dispositivo enfatizaram que o narguilé não é menos prejudicial à saúde. Assim, tendo em vista que a amostra analisada é composta por estudantes da área da saúde, que possuem maior acesso à informação quando comparados com o resto da população, era realmente de se esperar que os participantes possuíssem consciência de que o narguilé é prejudicial à saúde. No entanto, um dado alarmante é que, mesmo tendo conhecimento sobre os malefícios do instrumento, ainda fazem o seu uso. Ressalta-se, assim, que os atrativos ao uso extrapolam até mesmo o possível efeito lesivo do dispositivo, sugerindo um potencial de dependência e toxicidade (ABDULRASHID OA, et al., 2018).

Ao comparar este trabalho ao de Oliveira LAS (2016), percebeu-se que há um elevado uso de narguilé entre estudantes de medicina na região central goiana, sendo esses números semelhantes aos relatados em escolas médicas na Inglaterra $(51,7 \%)$, no Canadá (40\%), e na África do Sul (43,5\%), com proporções próximas (VANDERHOEK AJ, 2018). Estes números evidenciam que, mesmo com políticas de saúde, conhecimento médico e prática clínica, os participantes da pesquisa não assumem modelos exemplares para seus pacientes e para a população em geral, ao contrário, a cada ano a prevalência tende a aumentar devido aos motivos supracitados (MALTA DC, 2020).

Nesse sentido, sabe-se que com o crescente aumento do consumo de narguilé evidenciado, o cenário atual é altamente preocupante, pois os profissionais de saúde deveriam não somente aconselhar seus pacientes a manter práticas saudáveis, mas também servir de modelo de conduta, pois dessa forma a abordagem médica seria mais eficaz (MARTINS SE, et al., 2018). 
Ainda, em ambos os estudos, nota-se similaridade quanto ao uso semanal, cujos valores apresentaram baixas porcentagens (OLIVEIRA LAS, 2016). Em contrapartida, a frequência de uso "esporádico" apresentou maiores percentuais. Tal resultado pode ter sofrido influência do período em que houve a coleta de dados, o qual coincidiu com a pandemia de COVID 19 (Coronavirus Disease 2019), onde as repercussões respiratórias da doença aliadas às medidas restritivas de reuniões e socialização podem ter reduzido o consumo recente (SILVA ALO, et al., 2020).

Nessa perspectiva, vale salientar que como o uso está relacionado à socialização promovida pela sua utilização e que envolve locais e companhias específicas, o resultado deste trabalho torna-se coerente ao momento atual. Contudo, este dado destaca a necessidade de alerta para o público jovem, que cada vez mais tem demonstrado tendência à cronificação dessas práticas em meios sociais, não restringindo-as somente ao período acadêmico, mas tendo-as como um hábito de vida persistente (TAMIM H, et al., 2020).

No que tange às limitações do trabalho, destaca-se um possível impacto da inserção da universidade na experimentação do narguilé como um fator relevante. Isto porque ao se comparar os dados obtidos por Oliveira LAS (2016) com aqueles desse estudo, ainda se nota grande prevalência de uso de narguilé entre os jovens universitários, embora não consiga correlacionar a influência da vida acadêmica no início do uso. Uma coorte prospectiva ou um estudo longitudinal poderiam apresentar respostas sobre o risco da vida universitária no contato com o narguilé.

Já quanto aos pontos fortes, ressalta-se número considerável de respostas válidas nos questionários apresentados, proporcionando à pesquisa mais confiabilidade e menor viés científico. Além disso, a comparação entre os resultados obtidos e os dados da literatura atual, que abrange o mesmo tema, demonstraram similaridade quanto às variáveis analisadas.

\section{CONSIDERAÇÕES FINAIS}

Por meio desta pesquisa, foi possível constatar que a maioria dos usuários de narguilé faz parte do público masculino, solteiro, com renda compreendendo a classe $\mathrm{C} 1$ e faixa etária maior que 30 anos. Quanto à caracterização do uso, a utilização do dispositivo foi predominantemente de modo esporádico, com aroma e sabor e sob influência de colegas, representando, assim, o fumo de cunho social. Destaca-se que a prevalência do uso de narguilé entre os futuros médicos é alta, ressaltando a necessidade de melhores programas de educação preventiva em universidades médicas, combatendo as chamadas fake news e formando profissionais que prezem por acabar com o ciclo do tabagismo, que perpassa as gerações.

\section{REFERÊNCIAS}

1. ABDULRASHID OA, et al. Factors contributing to the upsurge of water-pipe tobacco smoking among Saudi females in selected Jeddah cafés and restaurants: A mixed method study. Journal Family Community Medicine, 2018; 1(25): 1319.

2. ALMEIDA LM, et al. Névoas, vapores e outras volatilidades ilusórias dos cigarros eletrônicos. Cadernos de Saúde Pública, 2017; 33(3): 125-137.

3. ARAÚJO RS, et al. Fatores relacionados ao consumo do narguilé entre estudantes de medicina. Jornal Brasileiro de Pneumologia, 2019; 45(5): 48-53.

4. BARRENECHEA MA, et al. Prevalencia del consumo de tabaco en adolescentes. Influencia del entorno familiar. Revista Anais pediatria, 2017; 66(4): 357-366.

5. BARRETO SM, et al. Experimentação e uso atual de cigarro e outros produtos do tabaco entre escolares nas capitais brasileiras (PeNSE 2012). Revista Brasileira de Epidemiologia, 2018; 17(1): 62-76.

6. BERTONI N, et al. Electronic cigarettes and narghile users in Brazil: Do they differ from cigarettes smokers? Addict Behav, 2019; 106007(98): 1-9.

7. BRASIL. Lei n. 12.546, de 2011, regulamentada em 31 de maio de 2014. Proíbe o uso de cigarros, cigarrilhas, cachimbos e outros tabacos em locais coletivos. 2014. Disponível em: https://jus.com.br/artigos/23875/brevesconsideracoes-acerca-do-recolhimento-previdenciario-misto-trazido-pela-lei-n-12-546-2011. Acessado em: 22 de maio de 2020.

8. FONSECA A. Tabaco e Tabaquistas. Arquivos de Medicina, 2017; 21(5): 183-193. 
9. HORTA BL, et al. Tabagismo em adolescentes de área urbana na região Sul do Brasil. Revista Saúde Pública, 2021; 35(1): 159-164.

10. INSTITUTO NACIONAL DE CÂNCER (INCA). Ministério da Saúde, Secretaria de Políticas de Saúde. Programa Nacional do Controle do Tabagismo. 2019. Disponível em: https://www.inca.gov.br/programa-nacional-de-controle-dotabagismo/publicacoes. Acessado em: 22 de maio de 2020.

11. INSTITUTO NACIONAL DE CÂNCER (INCA). Ministério da Saúde. Campanha Nacional de Combate ao Fumo. 2015. Disponível em: https://www.inca.gov.br/publicacoes/hotsites/hotsite-dia-nacional-decombate-ao-fumo-2015. Acessado em: 22 de maio de 2020.

12. LUNELLI ML, et al. Análise das condições pulmonares de discentes tabagistas de cigarro e tabagistas de narguilé do Centro de Ciências da Saúde da Universidade Regional de Blumenau. Assobrafir Ciência, 2019; 7(1): 43-57.

13. MALTA DC, et al. Fatores associados ao uso de narguilé e outros produtos do tabaco entre escolares, Brasil 2015. Revista Brasileira de Epidemiologia, 2018; 21(1): 1-15.

14. MARTINS SE, et al. Experimentação de conhecimento sobre narguilé entre estudantes de medicina de uma importante universidade do Brasil. Jornal Brasileiro de Pneumologia, 2018; 40(2): 102-110.

15. MARTINS RS, SANTOS UP. Narguilé, uma forma de consumo de tabaco em ascensão. Jornal Brasileiro de Pneumologia, 2019; 45(5): 315-316.

16. MAZIAK W, et al. The global epidemiology of waterpipe smoking. Tobacco Control, 2019; 24(3): 3-12.

17. MONTEIRO CA, et al. Population-based evidence of a strong decline in the prevalence of smokers in Brazil (1989 2003). Bulletin of the World Health Organization, 2007; 85(5): 527-534.

18. OLIVEIRA LAS. Experimentação e uso de cigarro eletrônico e narguilé entre universitários. 2016. 90 f. Tese (Mestrado em Ciências da Saúde) - Programa de Pós-Graduação em Ciências da Saúde, Universidade Federal de Goiás, Goiânia, 2016.

19. OLIVEIRA MA. Uso e conhecimento do Narguilé entre estudantes universitários e fatores associados. 2019. Dissertação (Mestrado em Ciências da Saúde) - Universidade Estadual de Ponta Grossa, Ponta Grossa, 2019.

20. PEREIRA JS, et al. Prevalence of smokers among students of an institution of higher education in the backlands of Paraiba. Revista de Pesquisa: Cuidado é fundamental Online, 2018; 2(5): 3856-3863.

21. PEUKER AC, et al. Expectativas e beber problemático entre universitários. Psicologia: Teoria e Pesquisa, 2020; 22(2): 193-200.

22. REIS AAC, et al. Desafios para as políticas públicas voltadas à adolescência e juventude a partir da Pesquisa Nacional de Saúde do Escolar (PeNSE). Ciência \& Saúde Coletiva, 2018; 23(9): 2879-89.

23. RIBEIRO M, CRUZ RC. Jovens e o uso do narguilé: a saúde pode ser comprometida? Assobrafir Ciência, 2016; 7(1): 7-10.

24. SAADE G, et al. Patterns of tobacco use: results from the 2005 Global Youth Tobacco Survey in Lebanon. Eastern Mediterranean Health Journal, 2019; 6(12): 1280-1290.

25. SANTOS PU. Cigarro eletrônico - repaginação e renovação da indústria do tabagismo. Jornal Brasileiro de Pneumologia, 2018; 44(5): 345-346.

26. SILVA ALO, et al. COVID-19 and smoking: a high-risk association. Cadernos de Saúde Pública, 2020; 36(5): 1-4.

27. SZKLO AS, et al. Perfil de consumo de outros produtos de tabaco fumado entre estudantes de três cidades brasileiras: há motivo de preocupação? Cadernos de Saúde Pública, 2021; 27(1): 2271-2275.

28. TAMIM H, et al. Tobacco use by university students, Lebanon, 2001. Addiction Journal, 2020; 98(7): 933-939.

29. TAVARES IB, et al. Prevalência e perfil de universitários que fumam narguilé no Distrito Federal. Programa de Iniciação Científica. Centro universitário de Brasília, Brasília, 2019.

30. TEIXEIRA L, JAQUES T. Legislação e controle do tabaco no Brasil entre o final do século XX e início do XXI. Revista Brasileira de Cancerologia, 2021; 57(3): 295-304.

31. TEODORO WR. Manejo do tabagismo na Atenção Básica. Dissertação (Curso de especialização em Atenção Básica em Saúde da Família) - Universidade Federal de Minas Gerais. Uberaba, 2019.

32. VANDERHOEK AJ, et al. An online survey of the habits, beliefs and knowledge base of medical students at a Canadian university. Tobacco Induced Diseases, 2018; 11(1): 9-14.

33. WARREN CW, et al. Change in tobacco use among 13-15 year olds between 1999 and 2008: findings from the Global Youth Tobacco Survey. Global Health Promotion, 2018; 16(2): 3890.

34. WERNECK FDA, et al. Prevalência do Tabagismo entre os estudantes de Medicina da Universidade Severino Sombra. Revista de Saúde, 2018; 7(2): 08-11.

35. WORLD HEALTH ORGANIZATION (WHO). Tobacco. 2020. Disponível em: https://www.who.int/news-room/factsheets/detail/tobacco. Acessado em: 03 de novembro de 2020. 